\title{
Correction to: On the ambiguous treatment of the Schrödinger equation for the infinite potential well and an alternative via singular potentials: the multi-dimensional case
}

\author{
Jesús Ildefonso Díaz ${ }^{1}$
}

Published online: 18 June 2018

(C) Sociedad Española de Matemática Aplicada 2018

\section{Correction to: SeMA (2017) 74:255-278 https://doi.org/10.1007/s40324-017-0115-3}

We show that the existence of flat solutions for the Schrödinger equation with super-singular potentials $V(x) \geq \underline{C} \delta(x)^{-2}$, with $\delta(x)=d(x, \partial \Omega)$, requires the condition $\underline{C}>N-1$. This corrects Theorem 2.1 of the mentioned paper by the author. New results and estimates are also presented.

One the main results of the paper [5] (which extends to the multidimensional case most of the conclusions of the previous paper by the author [4]) was to show that, given a bounded regular open set of $\mathbb{R}^{N}$, any eigenfunction $u_{n}$ of the problem

$$
D P(V, \lambda, \Omega) \begin{cases}-\Delta u+V(x) u=\lambda_{n} u & \text { in } \Omega, \\ u=0 & \text { on } \partial \Omega\end{cases}
$$

is a flat solution, in the sense that $\frac{\partial u_{n}}{\partial n}=0$ on $\partial \Omega$. This result was stated in Theorem 2.1 of [5] under the key assumption

$$
\frac{\underline{C}}{\delta(x)^{2}} \leq V(x) \leq \frac{\bar{C}}{\delta(x)^{2}} \quad \text { a.e. } x \in \Omega,
$$

for some $\bar{C}>\underline{C} \geq 0$. Here, $\delta(x)=d(x, \partial \Omega)$. Nevertheless one of the arguments of the proof must be corrected since the Moser iterative process, leading to the boundedness of $u_{n}$, does not imply (as a by-product) that $\delta^{-2} u_{n} \in L^{\infty}(\Omega)$ but merely that $u_{n} \in L^{\infty}\left(\Omega: \delta^{-2}\right)$. The first property means that

$$
\left\|\frac{u_{n}}{\delta^{2}}\right\|_{L^{\infty}(\Omega)}<+\infty
$$

The original article can be found online at https://doi.org/10.1007/s40324-017-0115-3.

Jesús Ildefonso Díaz

jidiaz@ucm.es

1 Departamento de Análisis Matemático y Matemática Aplicada, Instituto de Matemática Interdisciplinar, Universidad Complutense de Madrid, 28040 Madrid, Spain 
but it is not difficult to show (see, e. g., page 45 of [11]) that the second property is equivalent to the simpler fact that $u_{n} \in L^{\infty}(\Omega)$. Remember that after defining, for $1 \leq p<\infty$

$$
\|u\|_{L^{p}\left(\Omega: \delta^{-2}\right)}=\left[\int_{\Omega} \frac{|u(x)|^{p}}{\delta(x)^{2}} d x\right]^{1 / p},
$$

we have

$$
\lim _{p \nearrow+\infty}\|u\|_{L^{p}\left(\Omega: \delta^{-2}\right)}=\|u\|_{L^{\infty}\left(\Omega: \delta^{-2}\right)}=\|u\|_{L^{\infty}(\Omega)} .
$$

As a matter of fact, this slight mistake appeared already in the preprint of the paper [10] as I communicated to those authors. In spite of that, the main conclusion remains valid in the case of several dimensions under the additional condition $\underline{C}>N-1$ (notice that for $N=1$ this condition becomes merely $\underline{C}>0$ : as a matter of fact, the proof given in [4] for the one-dimensional case was entirely different to the one presented in [5]). Moreover, the quantitative estimate given in Theorem 2.1 of [5] should be slightly modified.

In the present new version, the key assumption will be generalized to the case of supersingular potentials $V(x)$ satisfying merely that

$$
\frac{C}{\delta(x)^{2}} \leq V(x) \text { a.e. } x \in \Omega,
$$

for some $\underline{C} \geq 0$ and thus it can be applied to show the existence of flat solutions for this class of potentials already considered in [7-9].

The following corrected version of Theorem 2.1 of [5] shows that the more general assumption (2), when $\underline{C}>N-1$, suffices to ensure that any eigenfunction $u_{n}$ is a flat solution. The existence of a countable set of eigenfunctions, under the general assumption (2), was shown in [7].

Theorem 2.1* Assume (2) and let $u_{n}$ be an eigenfunction of $D P\left(V, \lambda_{n}, \Omega\right)$ associated to the eigenvalue $\lambda_{n}$.

(a) There exists $\varepsilon \in[0,2), \varepsilon=\varepsilon(\underline{C}, N, n)$ and $\bar{K}_{n}=\bar{K}_{n}(\underline{C}, N, n, \Omega)>0$ such that

$$
\left|u_{n}(x)\right| \leq \bar{K}_{n} d(x, \partial \Omega)^{2-\epsilon} \text { a.e. } x \in \Omega \text {. }
$$

(b) If

$$
\underline{C}>N-1,
$$

then (3) holds for some $\varepsilon \in[0,1)$. In particular, $u_{n}$ is a flat solution.

(c) If

$$
\underline{C}>2 N
$$

then (3) holds for $\varepsilon=0$.

For the proof we shall need an auxiliary result constructing some suitable barrier functions (in the spirit of [4]) for the associated non-homogeneous Dirichlet problem

$$
\left(P_{f}\right) \begin{cases}-\Delta u+V(x) u=f(x) & \text { in } \Omega, \\ u=0 & \text { on } \partial \Omega .\end{cases}
$$

The existence, uniqueness and comparison principle of $u \in L^{1}(\Omega)$, very weak solution of problem $\left(P_{f}\right)$, when $f \in L_{\delta}^{1}(\Omega)$, under the general assumption that $V$ is nonnegative, was carried out in [7-9].

Lemma Let $f \in L_{\delta}^{1}(\Omega), V$ nonnegative, and let $u \in L^{1}(\Omega)$ be the (unique) very weak solution of the problem $\left(P_{f}\right)$. 
(i) Let $x_{0} \in \partial \Omega$ and assume

$$
V(x) \geq \underline{C}\left|x-x_{0}\right|^{-2} \text { with } \underline{C}>0 \text { a.e. } x \in \Omega
$$

and that there exists $R>0$ such that

$$
u \in L^{\infty}\left(\Omega_{R}\left(x_{0}\right)\right),
$$

where $\Omega_{R}\left(x_{0}\right):=\Omega \cap B_{R}\left(x_{0}\right)$. Let $\varphi(\varepsilon)=\varepsilon^{2}-\varepsilon(N+2)+2 N$, for $\varepsilon \in(0,2)$, and assume

$$
\varphi\left(\varepsilon^{*}\right)<\underline{C} \text { for some } \varepsilon^{*}=\varepsilon^{*}(\underline{C}, N) \in(0,2) .
$$

Assume that there exists $C_{x_{0}, R}>0$ such that

$$
f(x) \leq C_{x_{0}, R}\left|x-x_{0}\right|^{-\varepsilon^{*}} \text { a.e. } x \in \Omega_{R}\left(x_{0}\right) .
$$

Let

$$
C_{U}=C_{U, x_{0}, R, \varepsilon^{*}}=\max \left(\frac{C_{x_{0}, R}}{\underline{C}-\varphi\left(\varepsilon^{*}\right)}, \frac{\|u\|_{L^{\infty}\left(\Omega_{R}\left(x_{0}\right)\right)}}{R^{2-\varepsilon^{*}}}\right) .
$$

Then

$$
u(x) \leq C_{U}\left|x-x_{0}\right|^{2-\varepsilon^{*}} \text { a.e. } x \in \Omega_{R}\left(x_{0}\right) .
$$

(ii) Assume $f \in L^{\infty}(\Omega)$, (2) and (8), then, for any $x_{0} \in \partial \Omega$

$$
\frac{u(x)}{\left|x-x_{0}\right|^{2-\varepsilon^{*}}} \leq \frac{\|f\|_{L^{\infty}}}{\underline{C}-\varphi\left(\varepsilon^{*}\right)} M^{\varepsilon^{*}} \text { a.e. in } \Omega,
$$

for some $M=M(\Omega)>0$. In particular

$$
\frac{u}{\delta^{2-\varepsilon^{*}}} \in L^{\infty}(\Omega)
$$

(iii) Assume $f \in L^{\infty}(\Omega)$, (2) and (4), then estimate (13) holds for some $\varepsilon^{*} \in[0,1)$. In particular, $u$ is a flat solution.

(iv) Assume $f \in L^{\infty}(\Omega)$, (2) and (5), then estimate (13) holds for some $\varepsilon^{*}=0$.

(v) Assume (6), (5) and

$$
\psi\left(\beta^{*}\right)<\underline{C}, \text { for some } \beta^{*}=\beta^{*}(\underline{C}, N)>2,
$$

where $\psi(\beta)=\beta^{2}+\beta(N-2)$. Assume that there exists $R>0$ such that $u \in L^{\infty}\left(\Omega_{R}\left(x_{0}\right)\right)$ and that there exists $C_{x_{0}, R}>0$ such that

$$
f(x) \leq C_{x_{0}, R}\left|x-x_{0}\right|^{\beta^{*}-2} \quad \text { a.e. } x \in \Omega_{R}\left(x_{0}\right) .
$$

Let

$$
C_{U}=C_{U, x_{0}, R, \beta^{*}}=\max \left(\frac{C_{x_{0}, R}}{\left.\underline{C}-\psi\left(\beta^{*}\right)\right)}, \frac{\|u\|_{L^{\infty}\left(\Omega_{R}\left(x_{0}\right)\right)}}{R^{\beta^{*}}}\right) .
$$

Then,

$$
u(x) \leq C_{U}\left|x-x_{0}\right|^{\beta^{*}} \text { a.e. } x \in \Omega_{R}\left(x_{0}\right) .
$$

(vi) Assume (2), (5) and let $f \in L^{\infty}(\Omega)$ such that

$$
|f(x)| \leq C_{f} \delta(x)^{\beta^{*}-2} \text { a.e. } x \in \Omega,
$$

where $\beta^{*}>2$ is given by (14). Then

$$
|u(x)| \leq C_{U} \delta(x)^{\beta^{*}} \text { a.e. in } \Omega,
$$


with

$$
C_{U}=\frac{C_{f}}{\underline{C}-\psi\left(\beta^{*}\right)}
$$

In particular

$$
\frac{u}{\delta^{\beta^{*}}} \in L^{\infty}(\Omega)
$$

Proof (i) Let us consider the barrier function $U(x)=C_{U}\left|x-x_{0}\right|^{\beta}$ with $\beta>0$. Then, using spherical coordinates centered at $x_{0}$ we get

$$
\begin{aligned}
-\Delta U+V U & =(-\beta(\beta+N-2)+\underline{C}) C_{U}\left|x-x_{0}\right|^{\beta-2} \\
& \geq C_{f, x_{0}}\left|x-x_{0}\right|^{-\varepsilon} \geq f(x) \text { a.e. } x \in \Omega_{R}\left(x_{0}\right),
\end{aligned}
$$

once we assume

$$
\beta=2-\varepsilon^{*}
$$

and (10). Notice that $\beta(\beta+N-2)=\varphi\left(\varepsilon^{*}\right)$. Moreover, on $\partial \Omega_{R}\left(x_{0}\right) \cap \partial \Omega$ we trivially have $U \geq 0=u$. In addition, on $\partial \Omega_{R}\left(x_{0}\right) \backslash \partial \Omega$, thanks to (10), we have

$$
U(x)=C_{U} R^{2-\varepsilon^{*}} \geq\|u\|_{L^{\infty}\left(\Omega_{R}\left(x_{0}\right)\right)} \geq u(x) .
$$

Then, by the comparison principle we get that $u \leq U$ on $\Omega_{R}\left(x_{0}\right)$ which leads to (11).

(ii) Let us start by assuming that $f \geq 0$ a.e. on $\Omega$. Since $f \in L^{\infty}(\Omega)$ we have that $u \in L^{\infty}(\Omega)$ (see, e.g. [7,8]). We consider, again, the barrier function $U(x)=C_{U}\left|x-x_{0}\right|^{\beta}$ with $\beta=2-\varepsilon^{*}$, but now with

$$
C_{U}=\frac{\|f\|_{L^{\infty}}}{\underline{C}-\varphi\left(\varepsilon^{*}\right)} R\left(x_{0}\right)^{\varepsilon^{*}},
$$

where $R\left(x_{0}\right)=\max _{x \in \bar{\Omega}} d\left(x, x_{0}\right)$ (i.e. such that $\left.\Omega \subset B_{R}\left(x_{0}\right)\right)$. Then

$$
\begin{aligned}
-\Delta U+V U & \geq(\underline{C}-\varphi(\varepsilon)) C_{U}\left|x-x_{0}\right|^{-\varepsilon^{*}} \\
& \geq\left(\underline{C}-\varphi\left(\varepsilon^{*}\right)\right) C_{U} R\left(x_{0}\right)^{-\varepsilon^{*}} \\
& =\|f\|_{L^{\infty}(\Omega)} \text { a.e. in } \Omega .
\end{aligned}
$$

Since $U \geq 0=u$ on $\partial \Omega$ we have that $U \geq u$ a.e. in $\Omega$. This proves (12). In particular, since $\delta(x)=\min _{x_{0} \in \partial \Omega}\left|x-x_{0}\right|$ we have that

$$
\frac{u(x)}{\delta(x)^{2-\varepsilon^{*}}}=\max _{x_{0} \in \partial \Omega} \frac{u(x)}{\left|x-x_{0}\right|^{2-\varepsilon^{*}}} \leq \max _{x_{0} \in \partial \Omega} \frac{\|f\|_{L^{\infty}(\Omega)}}{\underline{C}-\varphi\left(\varepsilon^{*}\right)} M(\Omega)^{\varepsilon^{*}} .
$$

Here $M(\Omega)=\max _{x_{0} \in \partial \Omega} R\left(x_{0}\right)$, which is finite since $\Omega$ is a bounded set. This completes the proof of (13) when $f \geq 0$ a.e. on $\Omega$ (because then $u \geq 0$ a.e. on $\Omega$ ). In the general case, since $-f_{-} \leq f \leq f_{+}$, with $f_{+}(x)=\max \{f(x), 0\}, f_{-}(x)=\max \{-f(x), 0\}$, then we get that $u_{-} \leq u \leq u_{+}$where $u_{+} \geq 0$ (respectively $\left.u_{-} \leq 0\right)$ is the solution of $\left(P_{f_{+}}\right)$(respectively $\left.\left(P_{-f_{-}}\right)\right)$and the same arguments, applied now to $u_{-}$, leads to the conclusion.

Properties (iii), (iv) and (v) are simple consequences of the definition of $\varphi(\varepsilon)$ since $\varphi(\varepsilon)$ is a convex decreasing function such that $\varphi(0)=2 N, \varphi(1)=N-1$ and $\varphi(2)=0$.

For the proof of (v) and (vi) it suffices to consider the barrier function $U(x)=C_{U}\left|x-x_{0}\right|^{\beta^{*}}$ and to check that

$$
\begin{aligned}
-\Delta U+V U & =\left(\underline{C}-\psi\left(\beta^{*}\right)\right) C_{U}\left|x-x_{0}\right|^{\beta^{*}-2} \\
& \geq C_{f, x_{0}}\left|x-x_{0}\right|^{\beta^{*}-2} \geq f(x) \quad \text { a.e. } x \in \Omega_{R}\left(x_{0}\right),
\end{aligned}
$$


once we take $C_{U}$ given by (16). Notice that if $\beta=2-\varepsilon$ then $\psi(\beta)=\varphi(\varepsilon)$. To get (vi) it suffices to apply (v) to any point $x_{0} \in \partial \Omega$, since

$$
|f(x)| \leq C_{f} \delta(x)^{\beta^{*}-2} \leq C_{f}\left|x-x_{0}\right|^{\beta^{*}-2} \text { a.e. } x \in \Omega,
$$

and to use that $u \leq U$ on $\partial \Omega$ without any other additional condition on $C_{U}$.

Remark 1 We point out that we can get flat solutions even for suitable unbounded source functions $f(x)$. For instance, if we assume (4) and $f \in L_{\delta}^{1}(\Omega)$ satisfying (9) and $f$ bounded outside $\Omega_{R}\left(x_{0}\right)$ then the very weak solution is flat (some other examples are given in [2]).

Remark 2 Properties (v) and (vi) are typical of semilinear problems giving rise to a free boundary as for instance

$$
P\left(R, m, V_{0}, \lambda\right) \equiv \begin{cases}-\Delta v+V_{0}|v|^{m-1} v=f(x), & v \geq 0 \text { in } \Omega \\ v=0, & \text { on } \partial \Omega\end{cases}
$$

for a given $V_{0}>0$ and $m \in(0,1)$. In fact, this was the main argument used in the paper [4] to study flat solutions of $\left(P_{f}\right)$ (see, also, Section 3 of [5]) as well as [1] for the semilinear problem associated to super-singular potentials). Nevertheless, nothing similar to properties (i), (ii) and (iii) arise for such semilinear equations (except for the obstacle problem) if $f$ is not flat enough near $\partial \Omega$ (see, e.g. [3, Subsection 1.1c]).

Remark 3 In the case $\underline{C}=2 N$ the barrier function satisfies that $-\Delta U+V U=0$ and so it is a supersolution only when $f \leq 0$ a.e. on $\Omega_{R}\left(x_{0}\right)$. This could be applied to the study of the Signorini type problem for the equation $-\Delta u+V(x) u=f$ in a similar way to Theorem 2.50 of [3]).

Remark 4 Notice that, assumed that $V$ is bounded on any compact of $\Omega$, if $\underline{C}>2 N$ the regularity of the solution near the boundary $\partial \Omega$ is implied by the estimate with respect to $\delta(x)^{\beta(\underline{C}, N)-2}$ and, curiously enough, it depends (decreasingly with respect to the dimension $N$ of the space) on the value of the coefficient $\underline{C}$. For instance, if $\underline{C}=5$ then the solution is $C^{2}$ near $\partial \Omega$ for $N=1$ and $N=2$ but it is not so regular if $N \geq 3$. Some other results in this direction can be found in $[2,9,12]$ (see also [1] for the associated semilinear problem).

Remark 5 For pointwise arguments applied to the study of flat solutions in the context of relativistic (and fractionary) Schrödinger equations see [6,9].

Remark 6 For $0<\underline{C} \leq N-1$ the barrier function used in the proof of i) is an exact solution of a similar problem on $\Omega=\mathbb{R}^{N} \backslash\left\{x_{0}\right\}$ which is not flat on $\partial \Omega$. As a matter of fact, the flatness of the solution can appear merely over some subregions of $\partial \Omega$ (see [2]). It would be interesting to construct a general class of domains for which the associated solution is not flat in some subregion of $\partial \Omega$ for which (2) holds with $0<\underline{C}<N-1$ (see also some related results in this direction presented in [12]).

Proof of Theorem From Lemma 2.2 of [5], we know that $u_{n} \in L^{\infty}(\Omega)$. Then it suffices to apply, respectively, (ii), (iii) or (iv) of the above Lemma and the conclusions hold.

Remark 7 Some other results of the paper [5] are also affected by the mentioned correction (since they are consequence of its Theorem 2.1). It is the case of Corollary 2.1, Theorem 4.1 and Corollary 2.1 in which we must also assume the additional condition $C>N-1$. As a different minor modification, we point out that the exponent 2 in estimate (48) of [5] should be replaced by $2-\varepsilon$ if $\underline{C} \in(N-1,2 N)$. 
Acknowledgements Research partially supported by the project Ref. MTM2014-57113-P of the DGISPI (Spain) and the Research Group MOMAT (Ref. 910480) supported by the Universidad Complutense de Madrid.

\section{References}

1. Bandle, C., Pozio, M.A.: Sublinear elliptic problems with a Hardy potential. Nonlinear Anal. 119, 149-166 (2015)

2. Bernis, F., Díaz, J.I.: Local flat solutions for super-singular Schrödinger equations: the one dimensional case (in preparation)

3. Díaz, J.I.: Nonlinear Partial Differential Equations and Free Boundaries Research Notes in Mathematics, vol. 106. Pitman, London (1985)

4. Díaz, J.I.: On the ambiguous treatment of the Schrödinger equation for the infinite potential well and an alternative via flat solutions: the one-dimensional case. Interfaces Free Bound. 17, 333-351 (2015)

5. Díaz, J.I.: On the ambiguous treatment of the Schr ödinger equation for the infinite potential well and an alternative via singular potentials: the multi-dimensional case. SeMA J. 74(3), 255-278 (2017)

6. Díaz, J.I.: On the ambiguous treatment of the fractional quasi-relativistic Schrödinger equation for the infinite-well potential and an alternative via singular potentials. Main results presented in the Conference Nonlinear Partial Differential Equations and Mathematical Analysis, A workshop in the honor of Jean Michel Rakotoson for his 60th birthday, December 18-19, Madrid, Spain (2017) (in preparation)

7. Díaz, J.I., Gómez-Castro, D., Rakotoson, J.-M.: Existence and uniqueness of solutions of Schrödinger type stationary equations with very singular potentials without prescribing boundary conditions and some applications. Differ. Equ. Appl. 10(1), 47-74 (2018)

8. Díaz, J.I., Gómez-Castro, D., Rakotoson, J.M., Temam, R.: Linear equation with unbounded coefficients on the weighted space. Discrete Contin. Dyn. Syst. 38(2), 509-546 (2018)

9. Díaz, J. I., Gómez-Castro, D., Vázquez, J.L.: The fractional Schrödinger equation with general nonnegative potentials. The weighted space approach. Nonlinear Anal (2018). https://doi.org/10.1016/j.na.2018.05. 001. arXiv: 1804.08398

10. Drábek, P., Hernández, J.: Quasilinear eigenvalue problems with singular weights for the p-Laplacian. Annali di Matematica Pura ed Applicata

11. Opic, B., Kufner, A.: Hardy-Type Inequalities, Pitman Research Notes in Mathematics Series, vol. 279. Longman Scientific \& Technical, Harlow (1990)

12. Orsina, L., Ponce, A.C.: Hopf potentials for Schrödinger operators. Anal PDE 11(8), 2015-2047 (2018). https://doi.org/10.2140/apde.2018.11.2015 